\title{
Immunoglobulin G4-Related Hepatic Inflammatory Pseudotumor Diagnosed with Endoscopic Ultrasound-Guided Fine-Needle Biopsy
}

\author{
Akinobu Koiwai $^{a} \quad$ Morihisa Hirota $^{a}$ Mari Satoh $^{a}$ Atsuko Takasu $^{a}$ \\ Takayoshi Meguro $^{a}$ Katsuya Endo ${ }^{a}$ Takayuki Kogure ${ }^{a}$ Keigo Murakami ${ }^{b}$ \\ Kazuhiro Murakamib Kennichi Satoh ${ }^{\mathrm{a}}$ \\ aDivision of Gastroenterology, Tohoku Medical and Pharmaceutical University, Sendai, \\ Japan; ${ }^{b}$ Division of Pathology, Tohoku Medical and Pharmaceutical University, Sendai, Japan
}

\section{Keywords}

IgG4-related disease · Autoimmune pancreatitis · Inflammatory pseudotumor · Endoscopic ultrasound

\begin{abstract}
A 71-year-old man with obstructive jaundice was referred to our department. He underwent cholangiojejunostomy 15 years ago for palliative drainage. At that time, he had obstructive jaundice caused by an unresectable pancreatic head tumor. Contrast-enhanced computed tomography (CE$\mathrm{CT}$ ) now revealed a mass with low enhancement in the hepatic hilum that occluded the hilar bile duct and infiltrated extensively along the portal vein and hepatic artery. CE-CT also showed marked atrophy of the left hepatic lobe. No swelling or tumors were observed in the pancreas. Serum immunoglobulin G4 (lgG4) levels were as high as $465 \mathrm{mg} / \mathrm{dL}$. Endoscopic ultrasound-guided fineneedle biopsy (EUS-FNB) was performed targeting the hepatic hilar lesion. Immunohistological results of the biopsy specimens suggested that the lesion was an IgG4-related hepatic inflammatory pseudotumor (IPT) with no atypical cells. Steroid treatment resulted in rapid clinical improvement. This case suggested the usefulness of EUS-FNB for diagnosing IgG4-related hepatic hilar IPT.

(C) 2021 The Author(s).

Published by S. Karger AG, Basel
\end{abstract}

\section{Introduction}

Immunoglobulin G4-related disease (IgG4-RD) is a multi-organ immune-mediated condition that mimics many malignant, infectious, or inflammatory disorders. Histopathology is key to diagnosis [1]. IgG4-RD can sometimes manifest as inflammatory pseudotumors 
(IPTs) in various organs. IgG4-related IPTs are characterized histologically as irregular proliferation of myofibroblasts intermixed with infiltrates of inflammatory cells, mainly lymphocytes and plasma cells [2]. Hepatic IPTs are classified as lymphoplasmacytic or fibro-inflammatory based on clinical and histological features. Lymphoplasmacytic hepatic IPTs correspond to IgG4-RD and are always found around the hepatic hilum, especially around the hilar bile ducts [3]. The bile ducts adjacent to or within IgG4-related IPTs have cholangitis that resembles IgG4-related sclerosing cholangitis [3].

Clinical features and imaging findings of hepatic IPTs are sometimes similar to findings of intrahepatic or hilar cholangiocarcinoma. Some cases undergo surgical resection [4, 5]. Histological diagnosis is essential for differentiation from malignant tumors [3, 6], but it is often difficult to obtain sufficient tissue samples for diagnosis. Endoscopic ultrasound-guided fine-needle biopsy (EUS-FNB) is useful for differentiating IgG4-related hepatic hilar IPT from hilar cholangiocarcinoma.

\section{Case Report}

A 71-year-old man with obstructive jaundice was referred to our department. He had diabetes and no history of alcohol consumption or cigarette smoking. Approximately 15 years ago, when he was 56 years old, he was scheduled for pancreatoduodenectomy at another hospital due to a mass in the head of the pancreas, which had been preoperatively diagnosed as pancreatic ductal adenocarcinoma based on imaging findings. However, pancreatoduodenectomy was unsuccessful due to extensive tumor infiltration into the portal vein. The procedure consisted of only choledochojejunostomy for palliative bile duct drainage. The pathological diagnosis from an intraoperative tumor biopsy was not adenocarcinoma. The tumor in the head of the pancreas spontaneously resolved. At this time, laboratory tests showed high values of total bilirubin, $20.0 \mathrm{mg} / \mathrm{dL}$; direct bilirubin, $15.5 \mathrm{mg} / \mathrm{dL}$; aspartate aminotransferase, 43U/L; alanine aminotransferase, $82 \mathrm{U} / \mathrm{L}$; alkaline phosphatase, $336 \mathrm{U} / \mathrm{L}$; $\gamma$-glutamyl transpeptidase, $167 \mathrm{U} / \mathrm{L}$; and IgG4, $465 \mathrm{mg} / \mathrm{dL}$. White blood cell counts and levels of serum amylase and the tumor markers CEA, CA19-9, SPAN-1, and DUPAN-2 were within the normal range. Contrast-enhanced computed tomography (CE-CT) revealed a slightly enhanced mass occluding the hilar bile duct. The wedge-shaped tumor was located in the hepatic hilum and spread along the hepatic artery down to the superior edge of the pancreas. The portal vein was narrowed by the tumor, and there was well-developed collateral circulation. CE-CT imaging findings strongly suggested the tumor was advanced hilar cholangiocarcinoma with extensive infiltration of the hepatic artery and portal vein (Fig. 1a, b). CE-CT also showed marked atrophy of the left hepatic lobe. No swelling or tumors were found in the pancreas. The body and tail of the pancreas were atrophied and hypoenhancing on CE-CT (Fig. 1c). Magnetic resonance cholangiopancreatography revealed dilatation of the right intrahepatic bile duct, occlusion of the hilar hepatic duct, and no dilatation of the main pancreatic duct (Fig. 1d). On magnetic resonance imaging, the tumor in the hepatic hilum was hypointense on T1-weighted images. It infiltrated the hepatic hilum around Glisson's capsule. Although magnetic resonance imaging from approximately before 19 years showed swelling of both submandibular glands, no swelling of the salivary glands was observed at this time. Based on radiologic findings, high serum IgG4 levels, and his clinical history, the lesion was primarily considered to be IgG4-RD. Hilar cholangiocarcinoma was considered as part of the differential diagnosis.

Double-balloon endoscope-assisted endoscopic retrograde cholangiography (DB-ERC) was performed for cytological examination and biliary drainage. Although we reached the bile duct-jejunal anastomosis, only left intrahepatic bile ducts were visualized with contrast

\section{Karger's}



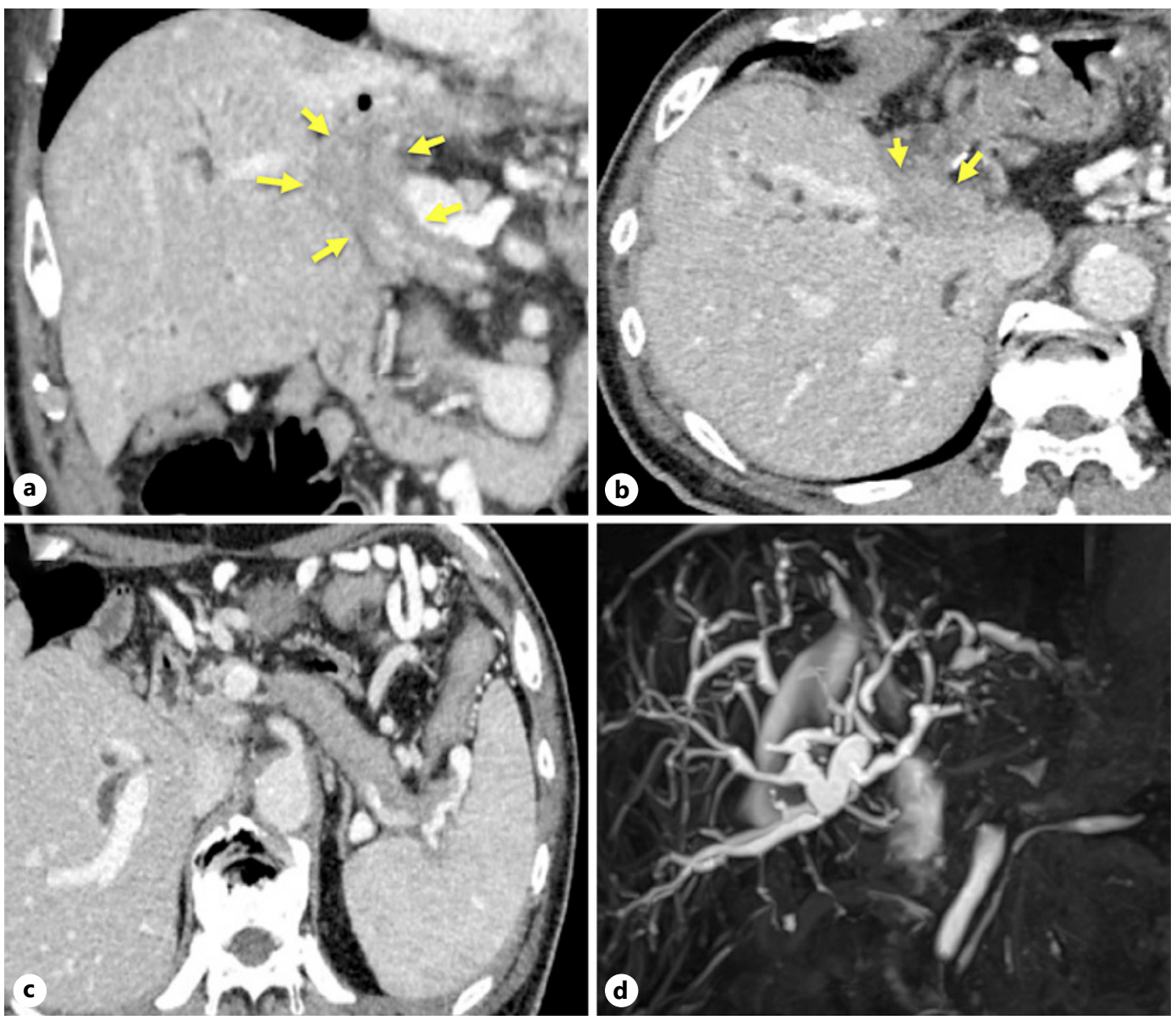

Fig. 1. Imaging findings of the wedge-shaped mass with low enhancement (yellow arrows) occluding the hilar bile duct. Coronal (a) and axial (b) abdominal CE-CT images. c No swelling or tumors were found in the pancreas. The body and tail of the pancreas were atrophied. d On MRCP, dilatation of the right intrahepatic bile duct and occlusion of hilar hepatic duct was observed, with no dilatation of the main pancreatic duct. CE-CT, contrast-enhanced computed tomography; MRCP, magnetic resonance cholangiopancreatography.

medium. It was not possible to pass a guide wire past the stenosis in the right hepatic duct. Both cytological examination and attempted biliary duct drainage of the right hepatic lobe were unsuccessful. Next, to exclude malignancy with a histological diagnosis, EUS-FNB was performed targeting the hypoechoic tumor in the hepatic hilum from the duodenal bulb using a convex scanning EUS with a 22G Franseen needle (Fig. 2a). During EUS-FNB, tissue specimens of the target lesion, including the bile duct and peribiliary glands, were collected. No atypical cells were observed.

Histopathology demonstrated dense lymphoplasmacytic infiltration and fibrosis (Fig. 2b), suspected obliterative phlebitis (Fig. 2c) and $>20$ IgG4-positive plasma cells per high-power field (Fig. 2d). Biopsy of the right lobe of the liver was performed because of elevated liver fibrosis markers, but no IgG4-positive plasma cells were observed in the liver parenchyma and peripheral bile ducts. No liver fibrosis was noted. Finally, we diagnosed the lesion in the hepatic hilum as an IgG4-related hepatic IPT based on the histopathological findings, high serum IgG4 values, and clinical history suggesting an asynchronous tumor with spontaneous resolution.

Prednisolone $(0.6 \mathrm{mg} / \mathrm{kg})$ was started. The dose was gradually decreased. After initial steroid treatment, the hepatic hilar IPT decreased in size and intrahepatic bile ducts became 

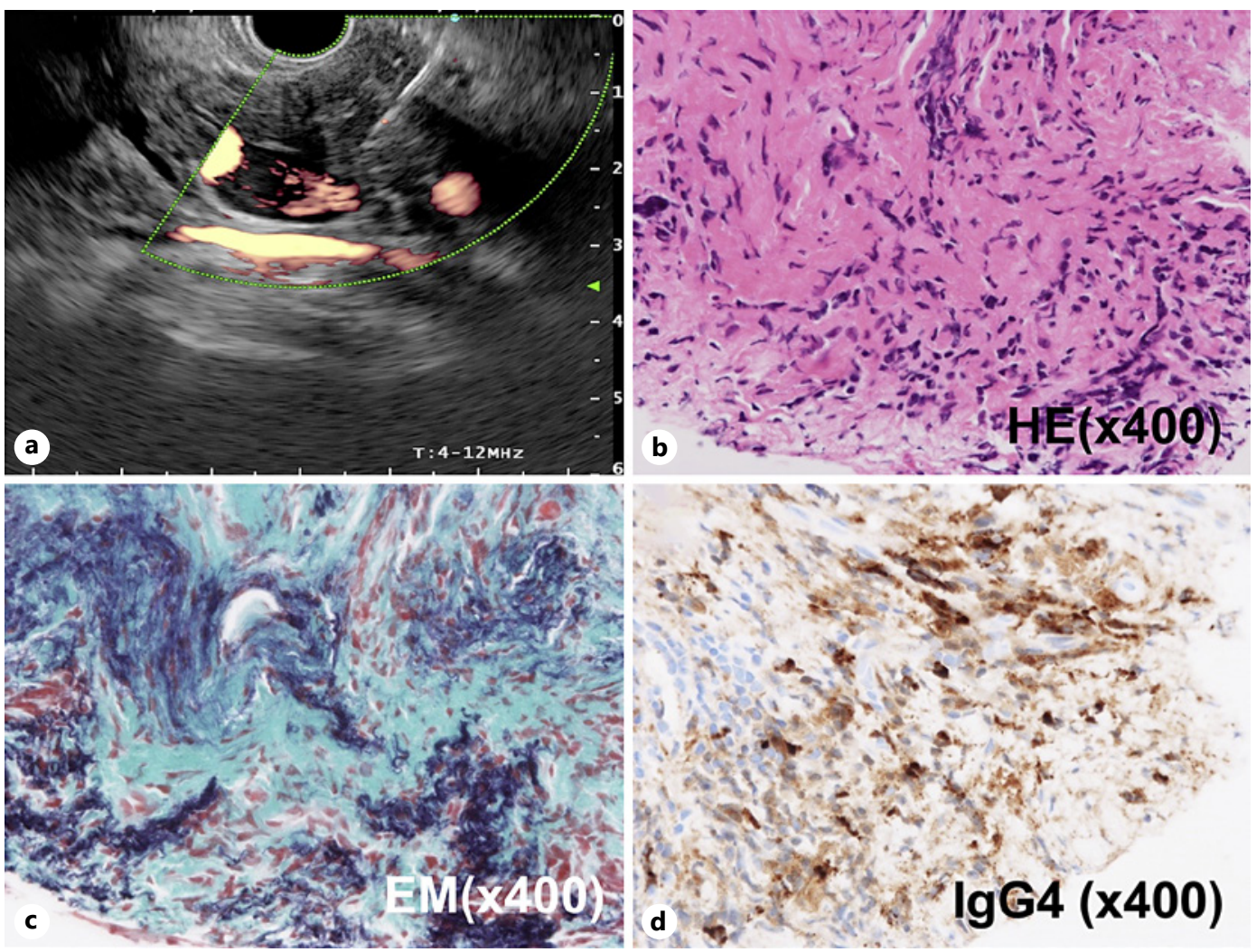

Fig. 2. a Lesion in the hepatic hilum was hypoechoic on EUS. EUS-FNB was performed from the duodenal bulb using a convex scanning EUS with a 22G Franseen needle. b HE staining of the biopsy sample showed dense lymphoplasmacytic infiltration and fibrosis in an HPF $(\times 400)$. c Obliterative phlebitis was suspected based on EM staining $(\times 400)$. d More than 20 IgG4-positive plasma cells were detected per HPF $(\times 400)$. EUS, endoscopic ultrasound; EUS-FNB, EUS-guided fine-needle biopsy; HE, hematoxylin and eosin; EM, elastica Masson; IgG4, immunoglobulin G4;HPF, high-power field.

less dilated (Fig. 3a, b). Total serum bilirubin and IgG4 values normalized (Fig. 3c). Seventeen weeks after the start of steroid therapy, maintenance prednisolone therapy $(5 \mathrm{mg} /$ day) was started without any problems.

\section{Discussion}

For the diagnosis of IgG4-RD, a consensus statement on the pathology of IgG4-RD and comprehensive diagnostic criteria for IgG4-RD have been published [6, 7]. However, depending on the location of the affected organ, it can be difficult to obtain a pathological sample. Recently, the 2019 American College of Rheumatology/European League Against Rheumatism Classification Criteria for IgG4-Related Disease was released [8]. The sensitivity and specificity for diagnosing IgG4-RD based on these criteria were $85.9 \%$ and $99.2 \%$, respectively. The criteria are divided into several steps (entry criteria, exclusion criteria, and inclusion criteria). IgG4-RD is diagnosed if items that meet inclusion criteria score 20 points or more. According to the latest diagnostic criteria, exclusion of malignancy is the most important prerequisite among the exclusion criteria, and histological findings are an important part of the inclusion criteria as well. It is necessary to obtain tissue samples for the diagnosis of IgG4-RD [9]. In this patient, imaging findings of the hepatic hilar lesion 


\section{Case Reports in Gastroenterology}
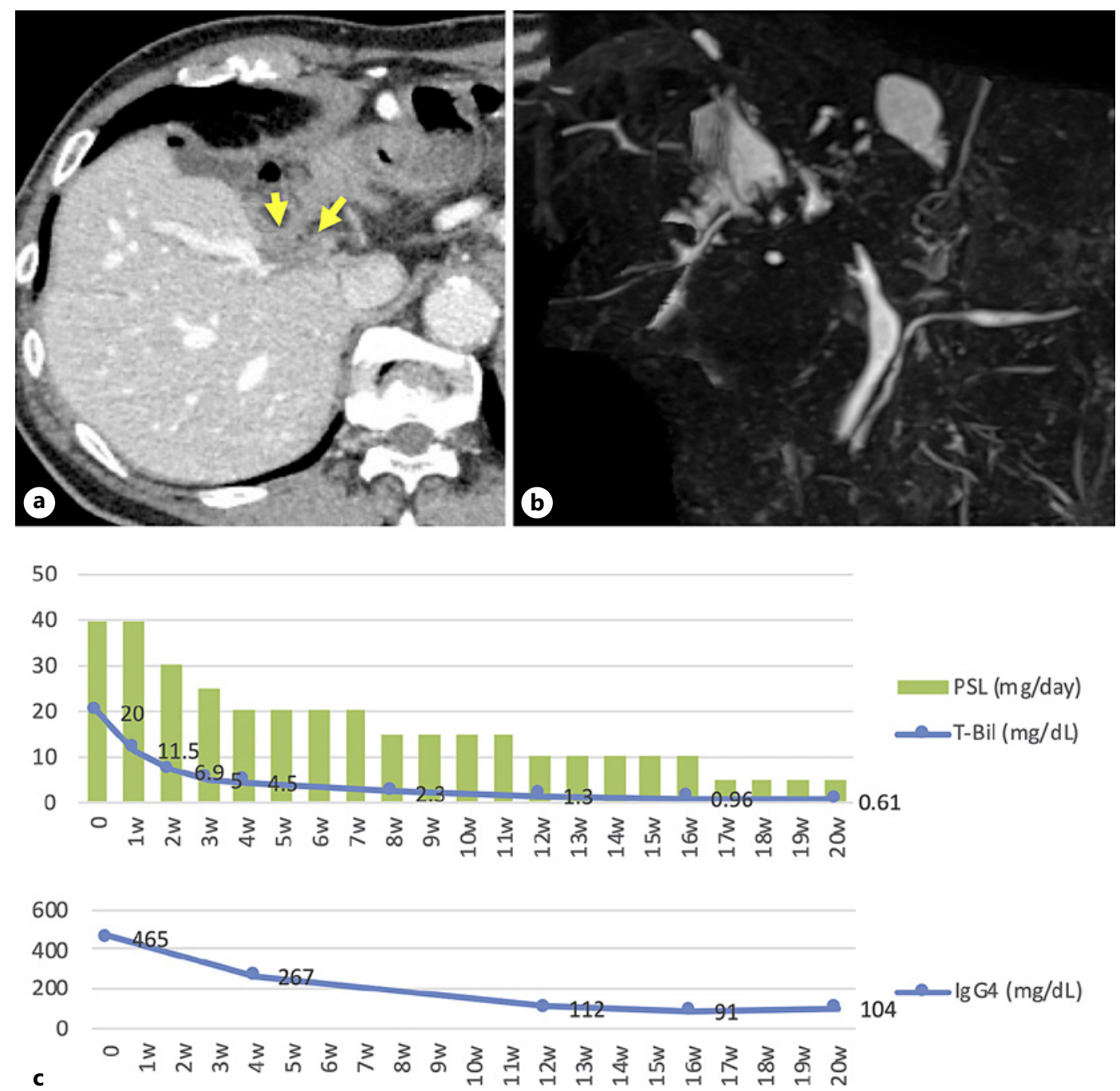

Fig. 3. a Twenty weeks after starting steroid treatment, CE-CT showed the hepatic IPT had decreased in size (yellow arrows). b MRCP showed less dilatation of the intrahepatic bile ducts. c After the start of steroid treatment, serum T-Bil and IgG4 values normalized. CE-CT, contrast-enhanced computed tomography; IPT, inflammatory pseudotumor; MRCP, magnetic resonance cholangiopancreatography; T-Bil, total bilirubin; IgG4, immunoglobulin G4; PSL, prednisolone.

were similar to those of advanced hilar cholangiocarcinoma with vascular infiltration. To exclude malignancy and confirm the pathological findings suggestive of IgG4-RD, it was necessary to perform a biopsy. Percutaneous liver biopsy targeting the legion in the hepatic hilum was difficult because of the distance from the body surface and the risk of puncturing blood vessels and bile ducts. We decided to perform EUS-FNB from the duodenal bulb, which was near the lesion, to obtain tissue samples. However, if the punctured lesion is cancer, needle tract seeding is a potential complication of needle biopsy $[10,11]$. Since IgG4-RD was considered to be the most likely diagnosis, despite the imaging findings, and this lesion was too advanced to be resected if it was malignant, we decided to perform EUS-FNB. According to a keyword search in PubMed, this was the first case in which IgG4related hepatic IPT was diagnosed with EUS-FNB. If this lesion was considered to have a high probability of being resectable hilar cholangiocarcinoma, surgery without EUS-FNB might have been an option. 
Steroid treatment for IgG4-RD is usually indicated for symptomatic patients. Asymptomatic patients are usually followed up without treatment. Organ dysfunction such as obstructive jaundice or renal failure is an absolute indication for treatment. Urgent and adequate treatment is required before the organ is irreversibly damaged [12]. In this case, marked obstructive jaundice due to severe hilar bile duct stenosis was an absolute indication for steroid treatment. Clinical practice guidelines for IgG4-related sclerosing cholangitis state that in cases involving obstructive jaundice due to biliary stenosis, biliary drainage is suggested before steroid administration [13]. Steroid treatment can be initiated without biliary drainage in patients with mild jaundice and no cholangitis for whom the diagnosis is definite, and pathologic diagnosis to establish the cause of biliary stenosis is unnecessary. As this patient had marked jaundice and no signs of infection, we attempted biliary drainage with DB-ERCP before starting steroid therapy, but it was unsuccessful. Bi et al. [14] reported that obstructive jaundice can be safely and effectively treated with steroids and no biliary stenting is required in patients with autoimmune pancreatitis, a type of IgG4-RD. In general, steroids are expected to have a high therapeutic effect in IgG4-RD [1]. In this patient, there were no signs of infection or contraindications to steroid therapy, and the general condition was good. Thus, we decided to start steroid treatment without biliary drainage. As expected, his obstructive jaundice decreased promptly (Fig. 3c). Maintenance steroid therapy was continued.

We encountered a patient with IgG4-related hepatic IPT that was difficult to distinguish from hilar cholangiocarcinoma with imaging workup alone. With EUS-FNB, we successfully obtained enough tissue from the lesion for immunohistological analysis, which was required for a definitive diagnosis of IgG4-RD. Obstructive jaundice in this case resolved with steroid treatment, without prior bile duct drainage. In conclusion, this report suggested that EUS-FNB is sometimes a useful method for diagnosing suspected IgG4-related IPT in the hepatic hilum.

\section{Statement of Ethics}

This study was reviewed and approved by the Institutional Review Board (approval number 2020-4-061). Written informed consent was obtained from the patient involved in the study for publication of this case report and any accompanying images.

\section{Conflict of Interest Statement}

The authors of this case report do not have any conflict of interest to declare.

\section{Funding Sources}

This case report has no funding sources.

\section{Author Contributions}

A.K. and M.H. prepared the first draft of the manuscript; A.K., M.H., M.S., A.T., and T.M. managed the patient; A.K. and M.H. performed endoscopic ultrasound-guided fine-needle biopsy and DB-ERCP; M.S. and T.K. performed liver biopsy; Ke.M. and Ka.M. performed pathologic evaluation; and K.E., T.K., and K.S. revised the manuscript. All authors approved the final version of the manuscript.

\section{Karger'}




\section{References}

1 Kamisawa T, Zen Y, Pillai S, Stone JH. IgG4-related disease. Lancet. 2015 Apr 11;385(9976):1460-71.

2 Kamisawa T, Okamoto A. IgG4-related sclerosing disease. World J Gastroenterol. 2008 Jul 7;14(25):3948-55.

3 Zen Y, Fujii T, Sato Y, Masuda S, Nakanuma Y. Pathological classification of hepatic inflammatory pseudotumor with respect to IgG4-related disease. Mod Pathol. 2007 Aug;20(8):884-94.

4 Kaneko K, Ando H, Watanabe Y, Seo T, Nagino M, Kamiya J, et al. Aggressive preoperative management and extended surgery for inflammatory pseudotumor involving the hepatic hilum in a child. Surgery. 2001 Jun; 129(6):757-60.

5 Naitoh I, Nakazawa T, Ohara H, Ando T, Hayashi K, Tanaka H, et al. IgG4-related hepatic inflammatory pseudotumor with sclerosing cholangitis: a case report and review or the literature. Cases J. 2009 Jun 11;2:7029.

6 Deshpande V, Zen Y, Chan JK, Yi EE, Sato Y, Yoshino T, et al. Consensus statement on the pathology of IgG4related disease. Mod Pathol. 2012 Sep;25(9):1181-92.

7 Umehara H, Okazaki K, Masaki Y, Kawano M, Yamamoto M, Saeki T, et al. Comprehensive diagnostic criteria for IgG4-related disease (IgG4-RD), 2011. Mod Rheumatol. 2012 Feb;22(1):21-30.

8 Wallace ZS, Naden RP, Chari S, Choi HK, Della-Torre E, Dicaire JF, et al. The 2019 American college of rheumatology/european league against rheumatism classification criteria for IgG4-related disease. Ann Rheum Dis. 2020 Jan;79(1):77-87.

9 Lévy S, Sauvanet A, Diebold MD, Marcus C, Da Costa N, Thiéfin G. Spontaneous regression of an inflammatory pseudotumor of the liver presenting as an obstructing malignant biliary tumor. Gastrointest Endosc. 2001 Mar;53(3):371-4.

10 Minaga K, Takenaka M, Katanuma A, Kitano M, Yamashita Y, Kamata K, et al. Needle tract seeding: an overlooked rare complication of endoscopic ultrasound-guided fine-needle aspiration. Oncology. 2017;93(Suppl 1):107-12.

11 Silva MA, Hegab B, Hyde C, Guo B, Buckels JA, Mirza DF. Needle track seeding following biopsy of liver lesions in the diagnosis of hepatocellular cancer: a systematic review and meta-analysis. Gut. 2008 Nov;57(11): 1592-6.

12 Khosroshahi A, Wallace ZS, Crowe JL, Akamizu T, Azumi A, Carruthers MN, et al. International consensus guidance statement on the management and treatment of IgG4-related disease. Arthritis Rheumatol. 2015 Jul; 67(7):1688-99.

13 Kamisawa T, Nakazawa T, Tazuma S, Zen Y, Tanaka A, Ohara H, et al. Clinical practice guidelines for IgG4related sclerosing cholangitis. J Hepatobiliary Pancreat Sci. 2019 Jan;26(1):9-42.

14 Bi Y, Hart PA, Law R, Clain JE, Farnell MB, Gleeson FC, et al. Obstructive jaundice in autoimmune pancreatitis can be safely treated with corticosteroids alone without biliary stenting. Pancreatology. 2016 May-Jun;16(3): 391-6. 